\title{
Surface quality control of a thin SiN layer by optical measurements
}

\author{
Jakub Gierowski, ${ }^{1,}$ Sandra Pawłowska, ${ }^{1 *}$ \\ ${ }^{I}$ Department of Metrology and Optoelectronics, Facultyof Electronics, Telecommunications and Informatics, \\ Gdańsk University ofTechnology, 1 1/12 Narutowicza Street, 80-233 Gdańsk, Poland
}

Received April 02, 2021; accepted September 08, 2021; published September 30, 2021

\begin{abstract}
Fiber optic interferometers have a wide range of applications, including biological and chemical measurements. Nevertheless, in the case of a reflective interferometer setup, standard silver mirrors cannot be used in every measurement, due to their chemical activity. This work investigates the surface quality of a thin optical layer of silicon nitride (SiN), which can serve as an alternative material for silver mirrors. We present measurements carried out with a Fabry-Perot fiber-optic interferometer working in a reflective mode. Measurement results allow us to determine the surface quality of the investigated layer.
\end{abstract}

The fiber optic configuration of an interferome ter has a lot of advantages, like ensuring stable and repeatable mea surements. Due to the application of op tic al fibers, small physical dimensions (hundreds of $\mu \mathrm{m}$ to $\mathrm{mm}$ ) of the measurement head can be achieved, which allows for their use in hard-to-reach places, performing nearly pointwise measurements. In addition, they are resistant to electromagnetic interference. They can be used in chemical and biological measurements [1]-[3]. An other advantage of using fiber optic interferometers is their lower cost in comparison to other sensors while their construction is rela tively simple.

The silver mirrors a re usually used as reflective la yers in interferometers. Such mirrors have high reflectivity and a wide range of reflected wa velengths, but their lif es p a n is short. Moreover, they can be easily mechanically da maged, so new materials to replace silver mirrors a re needed [4]. One of the possible materials can be amorphous silicon nitride ( $\mathrm{SiN}$ ) as it is more wearresistant [5]. It is also possible to apply it in biological measurements because $\mathrm{SiN}$ does not react chemically with biological samples [6].

Our goal wa s to investiga te the surface quality of a $\mathrm{SiN}$ la yer [7]. The layer consisted of amorphic Silicon nit ride and was $100 \mathrm{~nm}$ thick. It was deposited on a silicon wafer, a llowing us to conduct measurements u sing silicon as a main reflecting surface [8].

The measurement setup consisted of a light source (NKT Photonics EXR-20 Supercontinuum Laser with an adjustable optical filter, Denmark), single-m ode optical fibers, a 1x2 (50/50) fiber coupler (Cellco, Pola nd), a nd an optical spectrum a nalyzer (Anritsu MS9740a, Ja pan ). The measurement head wa s built to work as a Fabry-Perot

$$
{ }^{*} \text { E-mail: sandra.pawlowska@pg.edu.pl }
$$

interferometer operating in a reflective mode. Light from the light source was transmitted to the sensing interferometer through optical fibers and the fiber coupler. The signal from the interferometer was then transmitted back to the spectrum a nalyzer. A simplified mea surement setup schema is presented in Fig.1.

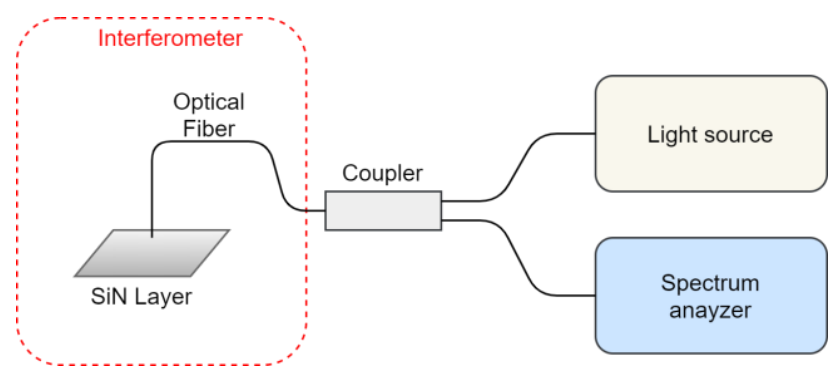

Fig. 1. Measurement setup.

The interferometer wa s built by placing an optical fiber end-face (acting a s a first reflecting surface) in parallel above the investigated $\mathrm{SiN}$ layer (acting as a second reflecting surface). The optical cavity between the reflecting surfaces was filled with air. The distance between the fiber end-face and the investigated la yer wa $\mathrm{s}$ set to $100 \mu \mathrm{m}$ by a micrometric screw controlling position of the fiber in the vertical a xis.

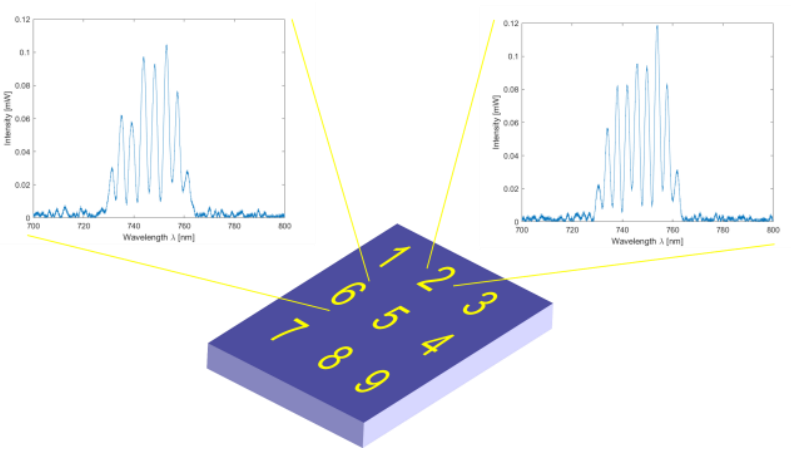

Fig. 2. Position of measurement points on a SiN layer (blue cuboid).

The sample surface quality was investigated by measuring spectra for nine chosen points at the surface of the SiN layer. The position of these points is shown in Fig. 2. The mea surement of each point was conducted in a series of 5 measurements to obtain statistical data. Each mea surement was made one after another with 15 second 
breaks between them and withoutchanging the position of the measuring head. This method provides that only source fluctuation and investigated layer changes impacted the result of measurement.

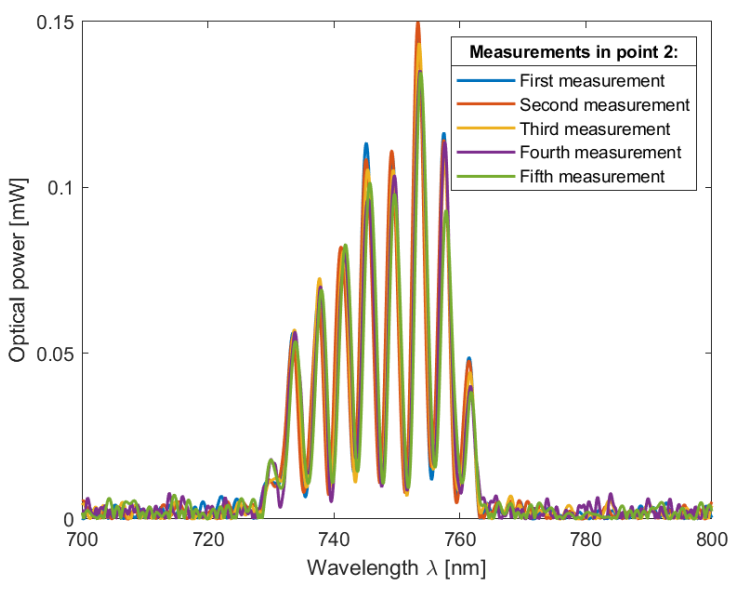

Fig. 3 Comparative signal spectrum for measured point 2.

Figure 3 shows five spectral cha racteristics. They were captured for the point 2 marked in Fig. 2 and investigated one by one. This method provides the opportunity to observe the change of signal and define surface quality of the $\mathrm{SiN}$ la yer. Spectral characteristics in Fig. 3 a re similar to each other, overlapping nearly perfectly. They have the same shape and the number of peaks in the spectrum remains stable in the following measurements. Maximum intensity of the signal and the corresponding central wavelength in each characteristic have changed in significantly. Thesechanges are caused by the source instability and are independent on the examined la yer. The only parameter shown in Fig. 3 that is dependent on the SiN layer is the positions of peaks in the measured spectrum.

The position statistics of a selected peak in the spectrum can be used to determinate surface quality. We decided to use a box plot technique. This method is used to show statistics of measurements with less data a vailable than in other methods, e.g., histogram. To use a box plot it is enough to havefive measurements for each point to receive reliable data [9]. Additionally, box plots allow to present data in a more transparent way, which makes it ea sier to compare them and draw conclu sions. The single box plot is a rectangle, which consists of three elements: lower quartile (25 percentile), median (50 percentile) and upper quartile ( 75 percentile). Between the lower and upper quartile is an interquartile range. This is a $50 \%$ of the central data. Quartiles are not sensitive to outliers, so they keep information a bout the center of data and distribution. The box plot can have whiskers which connect the rectangle with maximum or/and minimum value of data. If the outlier appears, it is shown individually. [9]

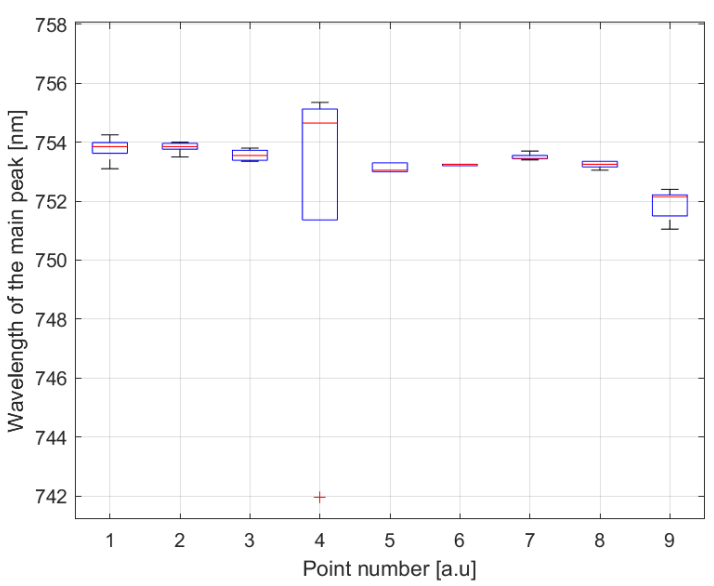

Fig. 4. Statistics of the wavelength of the main peak.

Figure 4 shows the position statistics of main peak in the measured spectra. It was calculated as the position of maximum closest to $754 \mathrm{~nm}$. Because the layer thickne ss should not vary in a significant way, the position of a selected peak should only shift by single na nometers or less. This can be observed in all points except points 4 and 9 . As they are positioned on the edge of an examined sample this can mean that this part was damaged. Layer thickness cannot be calculated from the position of a selected peak, but differences in thickness between the mea sured points can be calculated. To determine how a change in the layer thickness influences the position of a selected peak, computer simulation was used. The measured setup was simulated as a Fabry-Perot interferometer with three reflecting media boundaries: fiber-optic-air, a ir-SiN and SiN-Si. Ba sed on simulation results, estimated layer thickness distribution was calculated. Theresults were shown in Fig. 5.

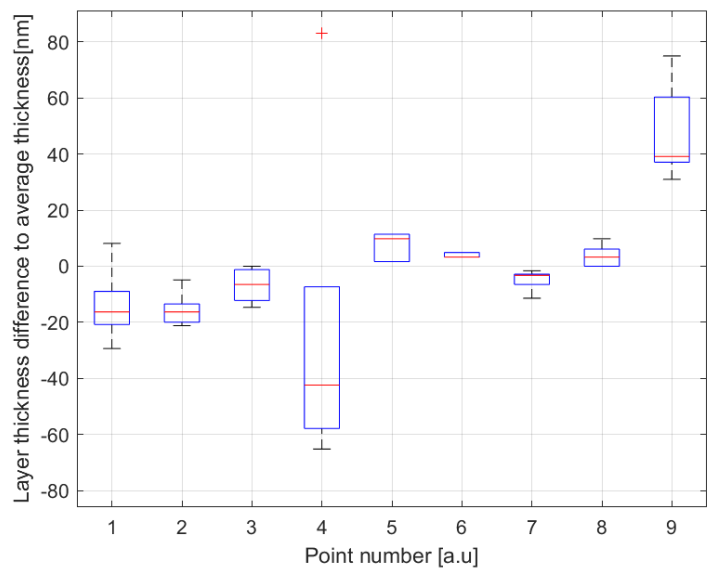

Fig. 5. Layer thickness distribution.

The results in Fig. 5 showed that the examined la yer has acceptable surface quality. In points 1,2 and 3 , the 
layer was thinner, while in 5, 6, 7 and 8 - thicker. As alrea dy mentioned, the results gathered from points 4 and 9 indicate the damage of the layer and the obtained thickness results ca n be different from real values.

Financial support of these studies from the Gdansk University of Technology in the frame of DEC1/2020/IDUB/III.4.3/PU grant under the Plutonium, DEC- 4/2020/IDUB/III.4.1/Tc and DEC8/2020/IDUB/III.4.1/Tc grant under the Technetium Talent Management Grants - 'Excellence Initiative Research University' Program is gratefully acknowledged. The authors want to acknowledge the financial support of DS funds of the Faculty of Electronics, Telecommunications, and Informatics of the Gdansk University of Technology and are gratefulfor the la ser rental of NKT Photonics from Interlab company. The authors would also like to thank Ph.D. Marcin Juchniewicz and M.Sc Bartłomiej Stonio for creating the $\mathrm{SiN}$ layerused for the research presented in this article.

\section{References}

[1] K. Karpienko, M.S. Wróbel, M. Jedrzejewska-Szczerska, Opt. Express 53, 077103 (2014).

[2] M. Jedrzejewska-Szczerska, M. Gnyba, B.B. Kosmowski, Acta Phys. Pol. A. 120, 621 (2011).

[3] M. Jedrzejewska-Szczerska, Sensors 14(4), 6965(2014).

[4] M. Kosowska, D. Majchrowicz, K.J. Sankaran, M. Ficek, K. Haenen, M. Szczerska, Materials 12, 2124 (2019).

[5] Shou-YiChang, Yi-Chung Huang, Microelectron. Eng. 84(2), 319 (2007).

[6] X. Wang, C. Wang, X. Shen, F. Sun, Appl. Opt. 56,4113 (2017).

[7] G. Coppola, P. Ferraro, M. Iodice, S. De Nicola, Appl. Opt. 42, 3882 (2003).

[8] H. Mäckel, R. Lüdemann, J. Appl. Phys. 92, 2602(2002).

[9] N. Atman, M. Krzywinski, Nat. Methods 11(2), 119 (2014).

[10] M. Vignesh, R. Balaji, International Conference on Innovations in Power and Advanced Computing Technologies (2017). 